\title{
Hydrofluoric acid etching of dental zirconia. Part 2: effect on flexural strength and ageing behavior.
}

\author{
Quentin Flamant ${ }^{1,2, *}$ and Marc Anglada ${ }^{1,2, *}$ \\ ${ }^{1}$ Department of Materials Science and Metallurgical Engineering, Universitat Politècnica de \\ Catalunya, Av. Diagonal 647, 08028 Barcelona, Spain. \\ ${ }^{2}$ Center for Research in Nano-Engineering, CRNE, Universitat Politecnica de Catalunya, C. \\ Pascual i Vila, 15, 08028 Barcelona, Spain. \\ * Corresponding Authors \\ E-mail: quentin.flamant@upc.edu \\ Phone number: +34 934054452 \\ E-mail: marc.j.anglada@upc.edu \\ Phone number: +34934016701 \\ Fax number: +34934016706
}

\begin{abstract}
Among the diverse treatments proposed to promote the osseointegration of zirconia dental implants, hydrofluoric acid (HF) etching appears to be a good candidate. However little is known on the effect of this process on the mechanical properties and long-term reliability. In this work, the surface integrity, the flexural strength and the ageing sensitivity of yttria-stabilized zirconia were assessed after etching in HF 40\%. Results show that etching induces an increase of monoclinic phase content and a decrease in flexural strength. The strength decrease is limited to $15 \%$ for etching times below $60 \mathrm{~min}$, whereas it reaches $29 \%$ after $120 \mathrm{~min}$ because of the formation of large etching pits. No substantial change in the ageing sensitivity was evidenced. Within the limits of this study, HF $40 \%$ etched zirconia appears to be reliable for long-term implantation provided that the etching duration does not exceed $60 \mathrm{~min}$.
\end{abstract}

Keywords: Zirconia, Etching, Dental, Ageing, Strength 


\section{Introduction}

Yttria-stabilized tetragonal zirconia polycrystals (Y-TZP, short: zirconia) are biocompatible and exhibit the best combination of strength and toughness of single-phase oxide ceramics. They were introduced as biomaterials in the end of the 1980s to overcome the limitations of alumina in the field of orthopedics [1]. Their excellent mechanical properties are due to phase transformation toughening: under stress the metastable tetragonal grains at the crack tip transform into monoclinic phase with a volume expansion, and this induces compressive stresses on the crack [2]. Unfortunately, the phase transformation is also the cause of their main weakness: in the presence of water the tetragonal phase can transform spontaneously, inducing micro-cracks and a loss of integrity of the material (see for instance the review from Lawson [3]). The impact of this phenomenon, known as Low Temperature Degradation (LTD) or ageing, was thought to be limited in vivo until 2001, when 400 Prozyr $^{\circledR}$ femoral heads failed in a very short period because of accelerated LTD [1]. This unfortunate event highlighted that minor changes in the fabrication process of zirconia ceramics may induce a dramatic increase of ageing sensitivity and that any modification should thus be followed by a careful assessment of its impact on long-term reliability.

While monolithic zirconia has been almost abandoned for orthopedic applications, in the last decade its use in restorative dentistry has been growing fast [4]. In particular, its good esthetics, high resistance to corrosion and the absence of allergic reaction make zirconia a good candidate to replace titanium for the fabrication of dental implants [5]. However, some authors reported a higher failure rate and a higher marginal bone loss when comparing zirconia to titanium. According to them, the use of zirconia implants does not appear recommendable at the moment 
except for specific cases (e.g. allergy to titanium), and there is a need for further research before generalizing their clinical use $[6,7]$.

To solve the problem of bone loss mentioned above, the key is to achieve a good osseointegration, which was shown to be promoted by rough surfaces [8]. Among the different surface chemical treatments already proposed in the literature for this purpose, hydrofluoric acid (HF) etching appears to be a good candidate [9-12]. Besides zirconia dental implants with acid etched surface are already commercialized (CeraRoot implants with ICETM surface) and apparently have shown a similar success rate as compared to Titanium implants after five years of follow-up study [13].

Despite the Prozyr ${ }^{\circledR}$ failure event, the dental industry may not have been sufficiently concerned with the problems related to ageing $[1,14]$. As a result, as far as the authors know, there is currently no study of the effect of acid etching on the long-term reliability of dental zirconia. The objective of this work is therefore to address this lack of knowledge by assessing the impact of HF etching on the surface integrity, the flexural strength and the ageing sensitivity. The surface characterization and the questions related to the etching mechanism are treated in a separated article [12].

\section{Materials and methods}

\subsection{Zirconia disks preparation}

Commercial 3Y-TZP powder (TZ-3YSB-E Tosoh Co., Japan) was cold isostatically compacted under pressure of $200 \mathrm{MPa}$ in a cylindrical mold for producing a green body, and then sintered in an alumina tube furnace at $1450{ }^{\circ} \mathrm{C}$ for two hours $\left(3{ }^{\circ} \mathrm{C} / \mathrm{min}\right.$ heating and cooling rates). The sintered ceramic cylinders were cut into specimens in the form of disks ( $2 \mathrm{~mm}$ thick, 
$9 \mathrm{~mm}$ diameter), which were ground and polished down to a $3 \mu \mathrm{m}$ diamond suspension. The samples were then successively cleaned for five minutes with acetone, ethanol and deionized water (DI water) in an ultrasonic bath in order to remove contaminants. The polishing step, which is not likely to be part of the processing for a commercial implant, was introduced in order to facilitate the study of the impact of etching on surface integrity, phase transformation and strength. It was assumed that the effects of etching on a machined and annealed surface or on a sintered surface would be comparable. Samples were divided into different groups according to the treatment they received as reported in Table 1 (ten samples per group).

Table 1. Names of the different groups of samples and description of the associated treatments.

\begin{tabular}{|c|c|}
\hline Group & Description of the treatment \\
\hline Control & No treatment (polished surface) \\
\hline HF40-30 & Immersed 30 min in HF $40 \%$ \\
\hline HF40-60 & Immersed 60 min in HF $40 \%$ \\
\hline HF40-120 & Immersed 120 min in HF $40 \%$ \\
\hline Control-Aged & Polished and aged \\
\hline HF40-30-Aged & Immersed 30 min in HF $40 \%$ and aged \\
\hline HF40-60-Aged & Immersed 60 min in HF $40 \%$ and aged \\
\hline HF40-120-Aged & Immersed 120 min in HF $40 \%$ and aged \\
\hline
\end{tabular}

\subsection{Chemical etching}

Etching was carried out in HF 40\% (Hydrofluoric Acid 40\% QP Panreac, Spain) and followed by ultrasonic cleaning in DI water. The volume of acid was $1 \mathrm{~mL}$ by sample. Etching times between zero and two hours and a concentration of $40 \%$ were used rather than longer times and more diluted solutions because these conditions were shown to be the most appropriate for a fast and uniform roughening [12]. 


\subsection{Surface integrity}

An estimation of the average thickness of the layer of material removed during etching was calculated using the following formula:

$$
t=\frac{\rho}{S} \Delta m(1)
$$

where $\mathrm{t}$ is the estimated removed thickness, $\Delta \mathrm{m}$ is the mass loss due to etching, $\rho$ is the theoretical density of 3Y-TZP $\left(\rho=6.1 \mathrm{~g} . \mathrm{cm}^{-3}\right)$ and S is the total sample external area.

The surface of etched samples was examined by Scanning Electron Microscopy (SEM) and the near-surface was observed on transversal sections milled with a Focused Ion Beam (FIB, Neon40, Carl Zeiss AG, Germany) in order to detect phase transformation and damage. Sample surfaces were protected with a thin platinum coating to flatten the surface and minimize ionbeam damage and curtain effect during milling. The final polishing of the cross-sections was performed at $500 \mathrm{pA}$.

\subsection{Ageing}

Hydrothermal degradation tests (which will also be referred to as "ageing") were performed in an autoclave, at $134^{\circ} \mathrm{C}, 100 \%$ steam atmosphere at $0.2 \mathrm{MPa}$ pressure during thirty hours. These conditions have been suggested to be equivalent to roughly sixty years in vivo for polished $3 \mathrm{Y}-\mathrm{TZP}[15]$

\subsection{Monoclinic phase content}

Three specimens of each group (Table 1) were analyzed by X-ray diffraction (XRD) with a Bruker AXS D8 diffractometer using $\mathrm{Cu}-\mathrm{K}_{\alpha}$ radiation to detect and quantify the tetragonal- 
monoclinic transformation. The monoclinic fraction was determined by using the relation proposed by Toraya et al. [16]:

$$
V_{m}=1.311 \frac{I_{m}(\overline{1} 11)+I_{m}(111)}{1.311\left[I_{m}(111)+I_{m}(111)+I_{t}(101)\right]}(2)
$$

where $\mathrm{V}_{\mathrm{m}}$ is the monoclinic volume fraction, $I_{m}(\overline{111})$ and $I_{m}(111)$ are the intensities of the monoclinic peaks and $I_{t}(101)$ is the intensity of the tetragonal peak.

\subsection{Biaxial flexural strength}

The biaxial flexural strength of the samples from the different groups (Table 1) was assessed by ball on three balls testing. The specimens were tested in an Instron 8511 servohydraulic fatigue testing machine with cobalt-cemented tungsten carbide balls of $5.9 \mathrm{~mm}$ diameter and a loading rate of $200 \mathrm{~N} / \mathrm{s}$. The fracture strength was calculated by using a numerical approximation of the maximum tensile stress as proposed by Börger et al. [17]:

$$
\sigma_{\max }=f \cdot \frac{F}{t^{2}}(3)
$$

where $\mathrm{F}$ is the applied load, $\mathrm{t}$ the sample thickness and $\mathrm{f}$ a dimensionless factor. Provided that

$0.55<\frac{R_{a}}{R}<0.9$ and $0.05<\frac{t}{R}<0.6$, an approximation of $\mathrm{f}$ for a determined Poisson's ratio can be calculated with the following formula:

$$
f=c_{0}+\frac{\left(c_{1}+c_{2} \frac{t}{R}+c_{3}\left(\frac{t}{R}\right)^{2}+c_{4}\left(\frac{t}{R}\right)^{3}\right)}{1+c_{5} \frac{t}{R}}\left(1+c_{6} \frac{R_{a}}{R}\right)
$$

where $\mathrm{R}$ is the radius of the disk, $\mathrm{R}_{\mathrm{a}}$ the support radius $\left(\mathrm{R}_{\mathrm{a}}=3.4 \mathrm{~mm}\right)$ and $\mathrm{c}_{\mathrm{i}}(\mathrm{i}=0, \ldots 6)$ tabulated constants which depend on the Poisson's ratio $v$ [17]. For 3Y-TZP, $v \approx 0.3$ [18], which leads to 
the following values: $\mathrm{c}_{0}=-17.346, \mathrm{c}_{1}=20.774, \mathrm{c}_{2}=622.62, \mathrm{c}_{3}=-76.879, \mathrm{c}_{4}=50.383, \mathrm{c}_{5}=$ $33.736, \mathrm{c}_{6}=0.0613$.

\subsection{Fractography}

The fractographic examination was a complicated task because samples were broken into tiny pieces. However for some of the specimens it was possible to identify the fracture origin by SEM.

\subsection{Statistical analysis}

Statistical analysis of the strength testing results was performed using SPSS ${ }$ software (version 20, SPSS Inc., Chicago, IL, USA). A two-way ANOVA with a 5\% significance level was used to evaluate the effects of etching time and ageing and to assess possible interaction. The homogeneity of variance between groups was verified with a Levene test. A simple main effects analysis with Sidak-adjusted $\alpha$ was applied to investigate specific comparisons.

The variability of the strength was analyzed using the Weibull distribution function:

$$
P_{F}(\sigma)=1-\mathrm{e}^{-\left(\frac{\sigma}{\sigma_{0}}\right) m}
$$

where $\mathrm{P}_{\mathrm{F}}$ is the probability of failure, $\sigma$ is the fracture strength, $\sigma_{0}$ is the Weibull characteristic strength, and $\mathrm{m}$ is the Weibull modulus.

For the graphical evaluation of $\mathrm{m}$ and $\sigma_{0}$ the measured strength data were ranked in increasing order and numbered from 1 to $\mathrm{N}$. Then the single strength values $\sigma_{\mathrm{i}}$ were related to the failure probability $\mathrm{P}_{\mathrm{Fi}}$ according to the following relation:

$$
P_{F i}=\frac{i-0.5}{N}(6)
$$


where $\mathrm{i}$ is the ranking number and $\mathrm{N}$ is the total number of measurements (for a more detailed description of the methodology, see for instance Munz et al. [19]). Finally, the $90 \%$ confidence bounds for $\mathrm{m}$ and $\sigma_{0}$ were determined according to ASTM C1239-00.

\section{Results}

\subsection{Surface integrity after etching}
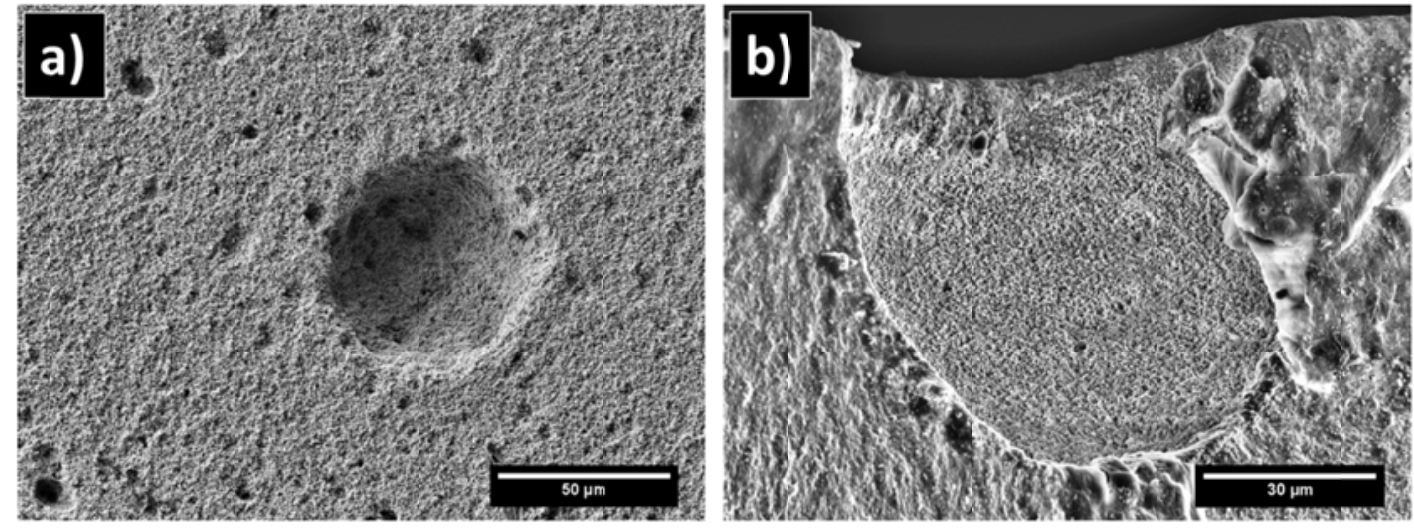

Figure 1. Pits of exceptionally big size observed: (a) on the surface of a sample from the HF40120 group, (b) on the fracture surface of a sample from the HF40-120-aged group, in which it nucleated failure $\left(\sigma_{\max }=816 \mathrm{MPa}\right)$.

The thickness of the layer of material removed during etching was estimated to be (mean value $\pm \mathrm{SD}$ ): $6.0 \pm 1.9 \mu \mathrm{m}, 12 \pm 1.0 \mu \mathrm{m}$ and $18 \pm 4.5 \mu \mathrm{m}$ for respectively 30,60 and 120 minutes of etching.

The observation of the FIB cross-sections evidenced that a small porosity was produced under the surface (Figure 2). The etch-damaged layer had an average thickness of about $1 \mu \mathrm{m}$, which reached locally $3 \mu \mathrm{m}$ but did not seem to increase substantially over time. 
On the other hand, etching produced numerous randomly dispersed pits on the surface, whose typical size increased with etching duration (Figure 3-b,c,d). The presence of pits of exceptionally big size was detected in few cases (Figure 1). As it will be discussed further, although this kind of defects was uncommon, they may have a critical impact on strength.
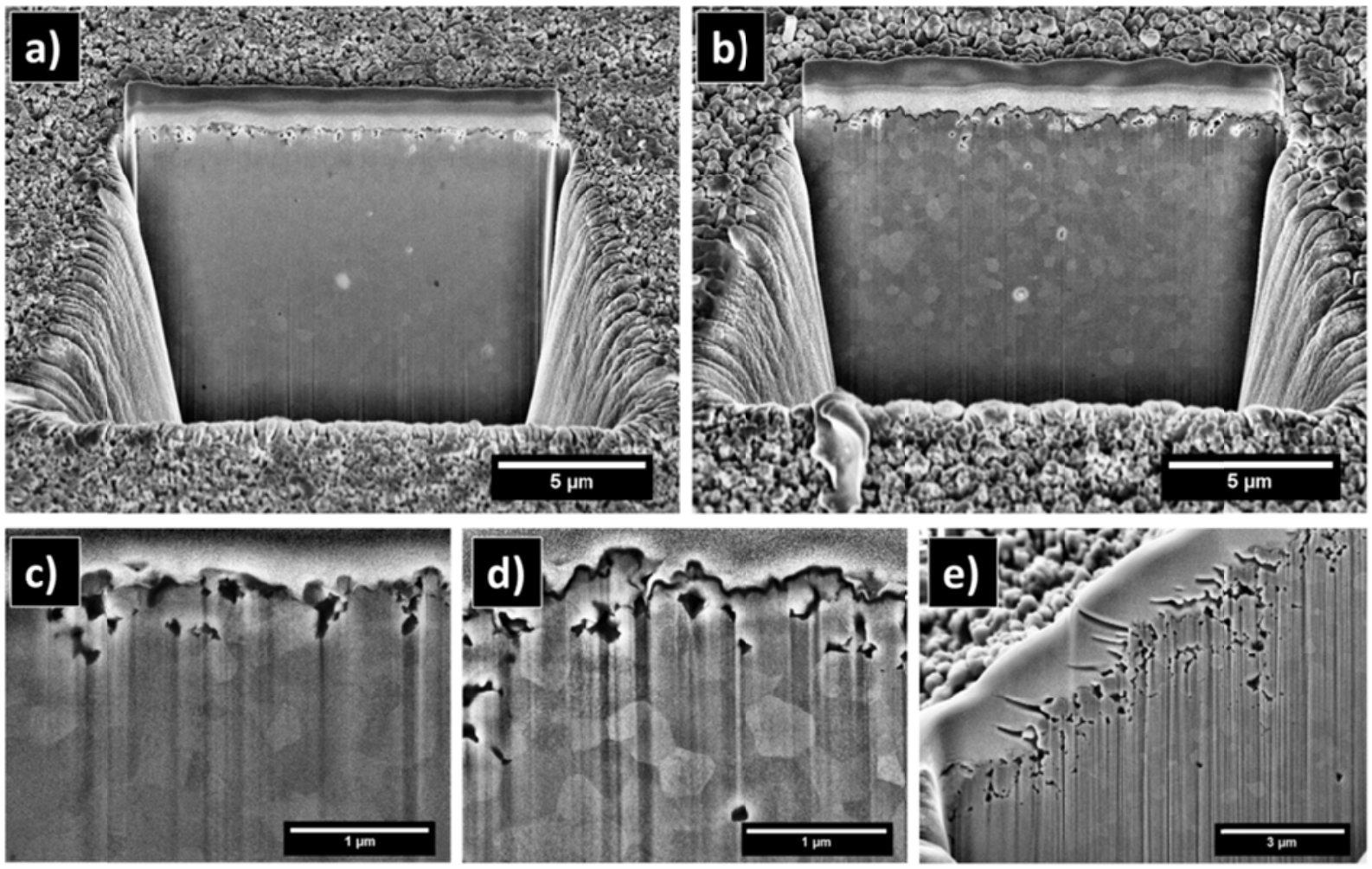

Figure 2. FIB cross-sections at the surface of $3 \mathrm{Y}-\mathrm{TZP}$ after etching for one hour $(\mathrm{a}, \mathrm{c})$ and two hours (b, d, e). 

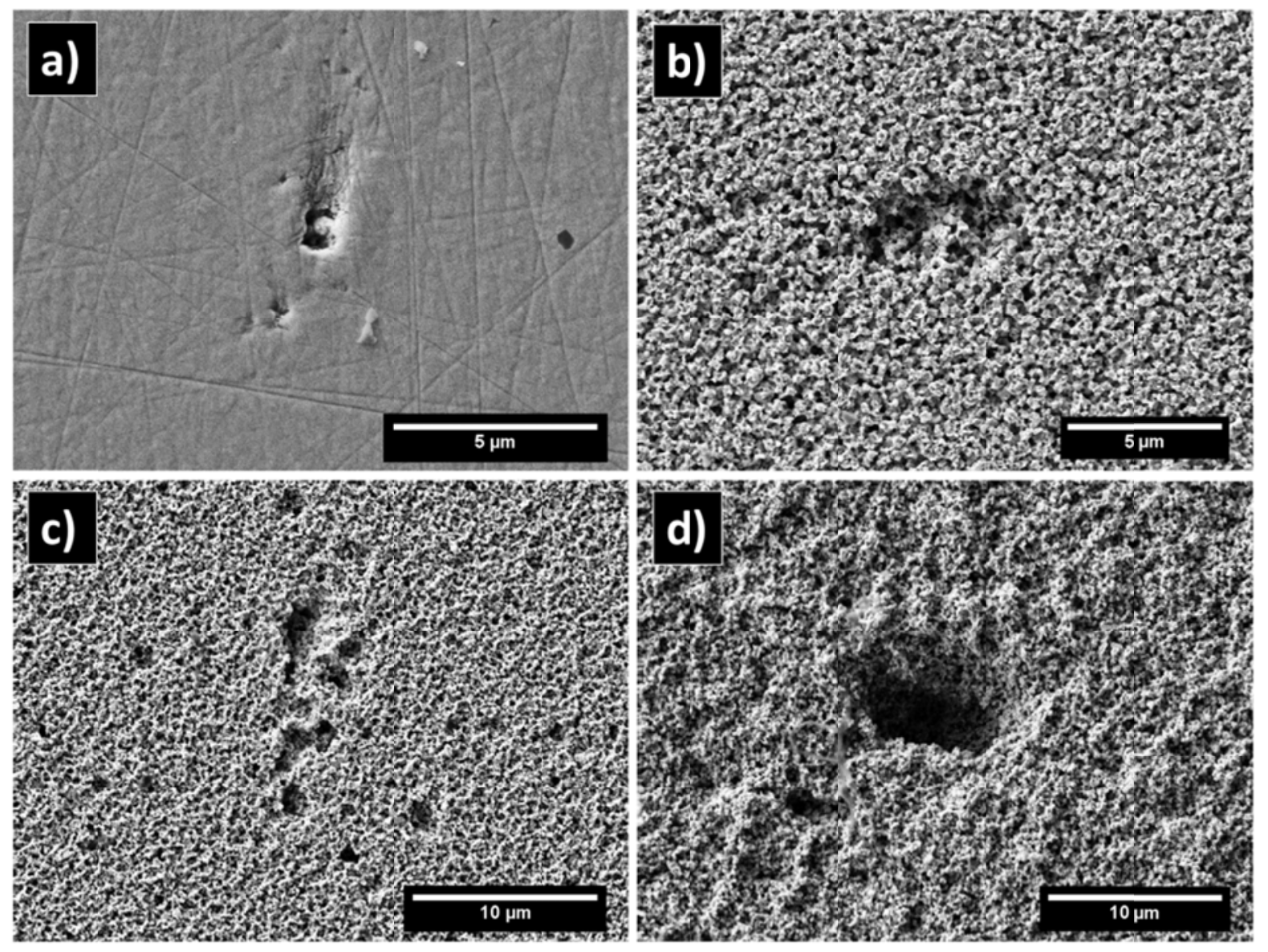

Figure 3. SEM observations of a pore on the polished surface (a), and typical pits after etching for: $30 \mathrm{~min}(\mathrm{~b}), 1 \mathrm{~h}(\mathrm{c}), 2 \mathrm{~h}(\mathrm{~d})$.

\subsection{Phase transformation}

Etching induced an increase of monoclinic phase content at the surface in the range 10-23\% nevertheless the results do not show a simple relation between this increase and etching time (Figure 4). On the other hand, the amount of monoclinic phase in samples of the different etchedaged groups (HF40-30-Aged, HF40-60-Aged, HF40-120-Aged) was very similar (in the range 71-73\%), indicating that the monoclinic content after ageing did not depend on the initial etching time. In the case of the Control-Aged group, the amount of monoclinic phase was lower (about 
$56 \%)$. However, the increase in monoclinic content $(+55 \%)$ was roughly the same as in the etched-aged sample groups (between $+48 \%$ and $+61 \%$ ). In other words, the difference in the final monoclinic content after ageing between the control-aged and the etched-aged samples was mainly associated to the difference in the initial amount of monoclinic phase.

Finally, the thickness of the micro-cracked layer induced by the phase transformation measured on a FIB cross-section of a sample from the HF40-120-Aged group was estimated to be $\mathrm{d} \approx 5 \mu \mathrm{m}$ (Figure 5). The same measurement performed on a sample from the Control-Aged group gave a similar result $(\approx 6 \mu \mathrm{m})$. This tends to confirm that etched samples do not age more rapidly than the control ones. 


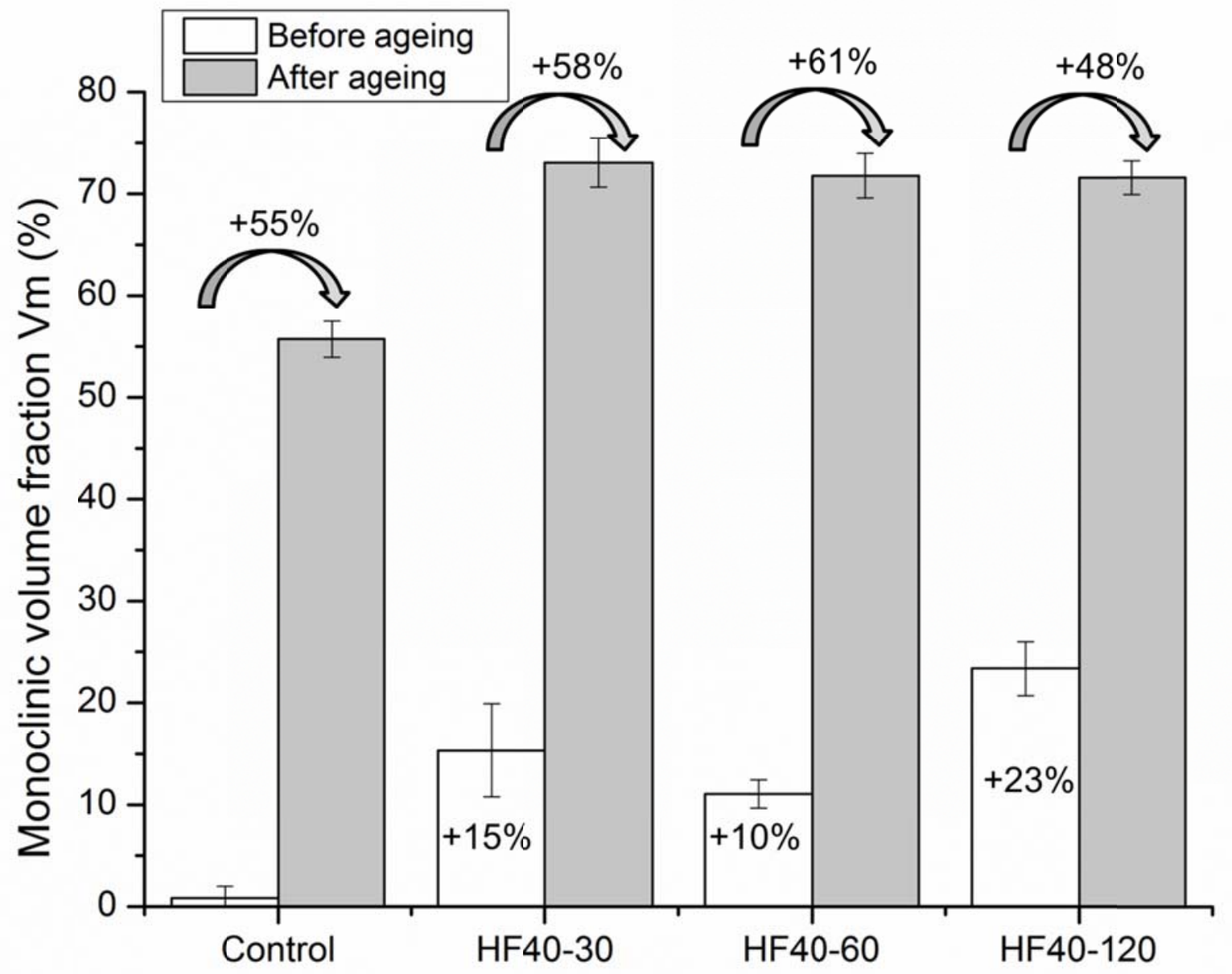

Figure 4. Monoclinic volume fraction determined from XRD measurements for different etching times, before and after ageing. Values inside the columns indicate the absolute variation with respect to the Control group. Values above the columns indicate the absolute variation after ageing. Error bars represent the standard deviations. 

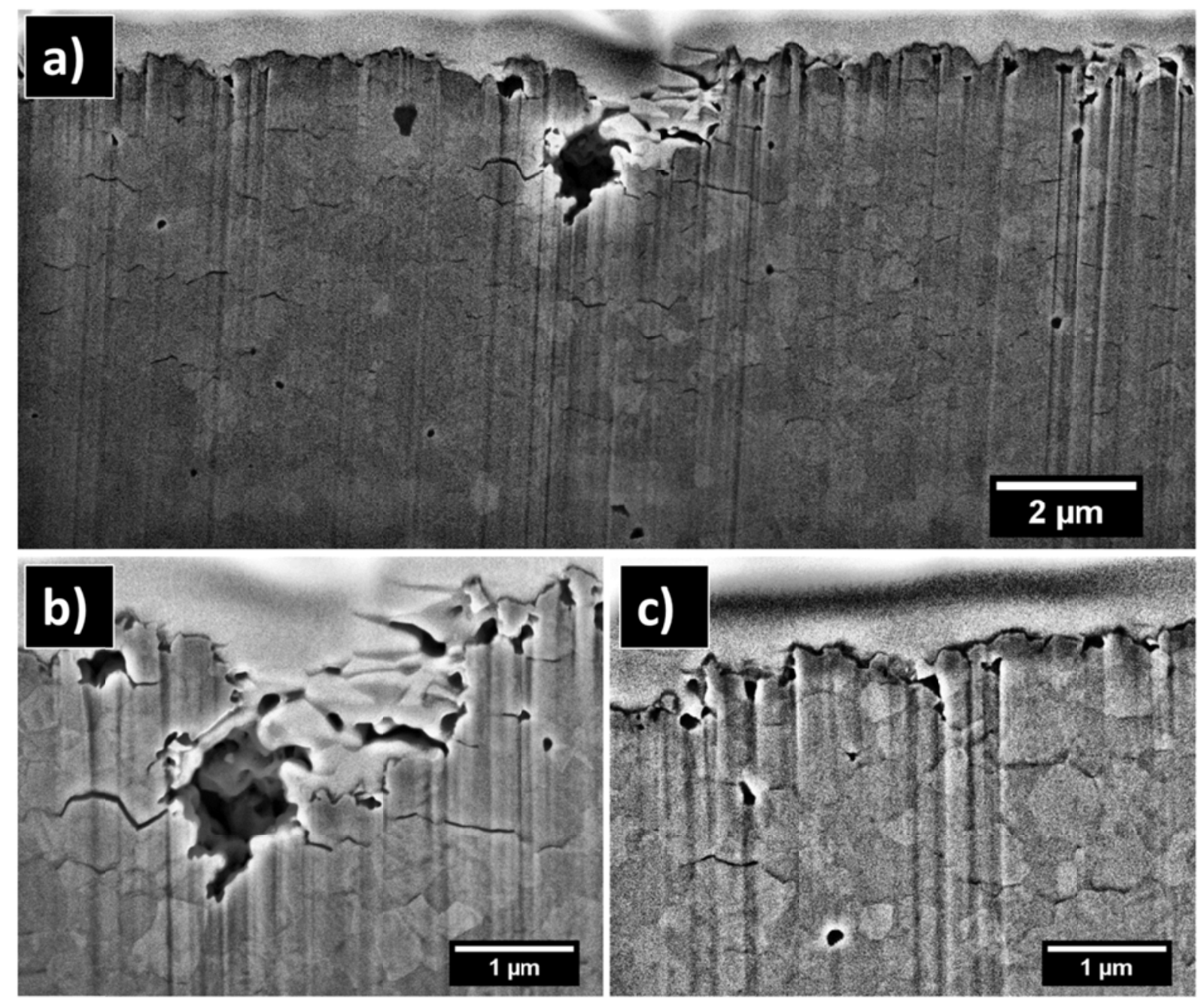

Figure 5. FIB cross-section at the surface of 3Y-TZP after etching for two hours and subsequent ageing: (a) full cross-section, (b) etching pit at high magnification, (c) micro-cracked zone at high magnification. 


\subsection{Fractography}

Fractographic observations could only be performed on a limited number of samples but evidenced that:

- Etching pits could initiate failure (Figure 1-b, Figure 6-b).

- Similarly, porous zones, which may result from a locally deeper penetration of the acid in the material, could initiate failure (Figure 6-a). These zones will be referred to as "deep porous zones".

- The monoclinic micro-cracked layer induced by ageing was thicker in some zones, which could also nucleate failure: for instance in the case presented Figure 6-c it reached $13 \mu \mathrm{m}$, which is approximately 2.5 times more than the average thickness.

One sample from each of the HF40-30 and HF40-60 groups had substantially lower strength than the rest because of very uncommon defects. In the first case the failure was associated to a "tunnel-like" defect (Figure 6-e). The acid seems to have removed preferentially the material under the surface, which could be due to some large pre-existing sub-superficial defect, like a pre-existing pore or a less densified zone. In the second case, the failure seems to have been initiated by a huge sub-superficial defect (Figure 6-f), but there was no evidence that it could be related to etching. Because these defects were very unusual and distinct from the others, the data points associated to those samples were discarded for the calculation of the mean strength and the standard deviation of the group they belonged to, as well as for the statistical analysis. 

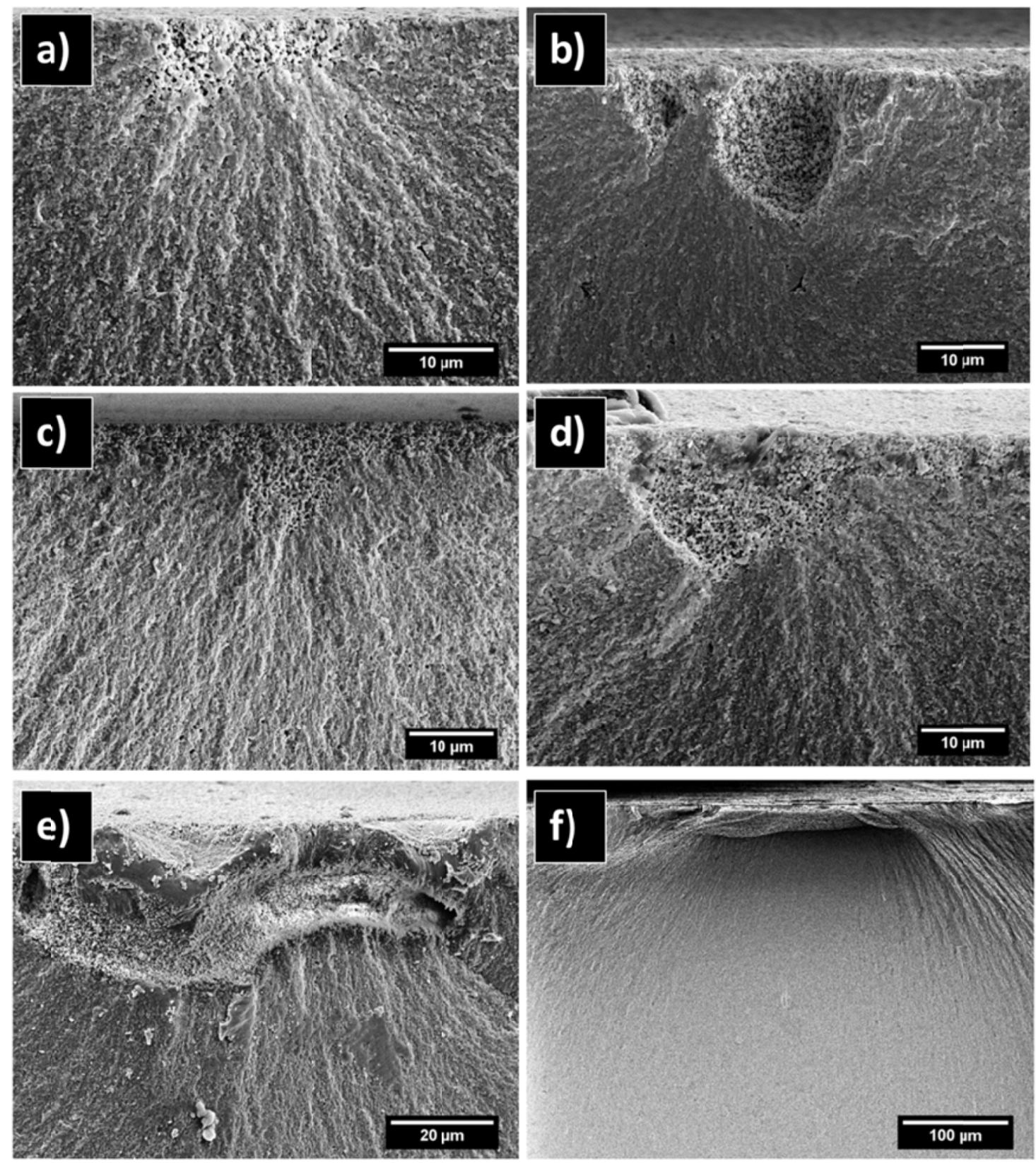

Figure 6. Observation by SEM of the failure origin of samples from: a) HF40-120 group $\left(\sigma_{\max }=1042 \mathrm{MPa}\right)$, b) HF40-120 group $\left.\left(\sigma_{\max }=732 \mathrm{MPa}\right), \mathrm{c}\right)$ Control-Aged group $\left(\sigma_{\max }=970 \mathrm{MPa}\right)$, d) HF40-120-aged group ( $\left.\sigma_{\max }=1146 \mathrm{MPa}\right)$, e) HF40-30 group $\left.\left(\sigma_{\max }=588 \mathrm{MPa}\right), \mathrm{f}\right)$ HF40-60 group $\left(\sigma_{\max }=359 \mathrm{MPa}\right)$. 


\subsection{Strength analysis}

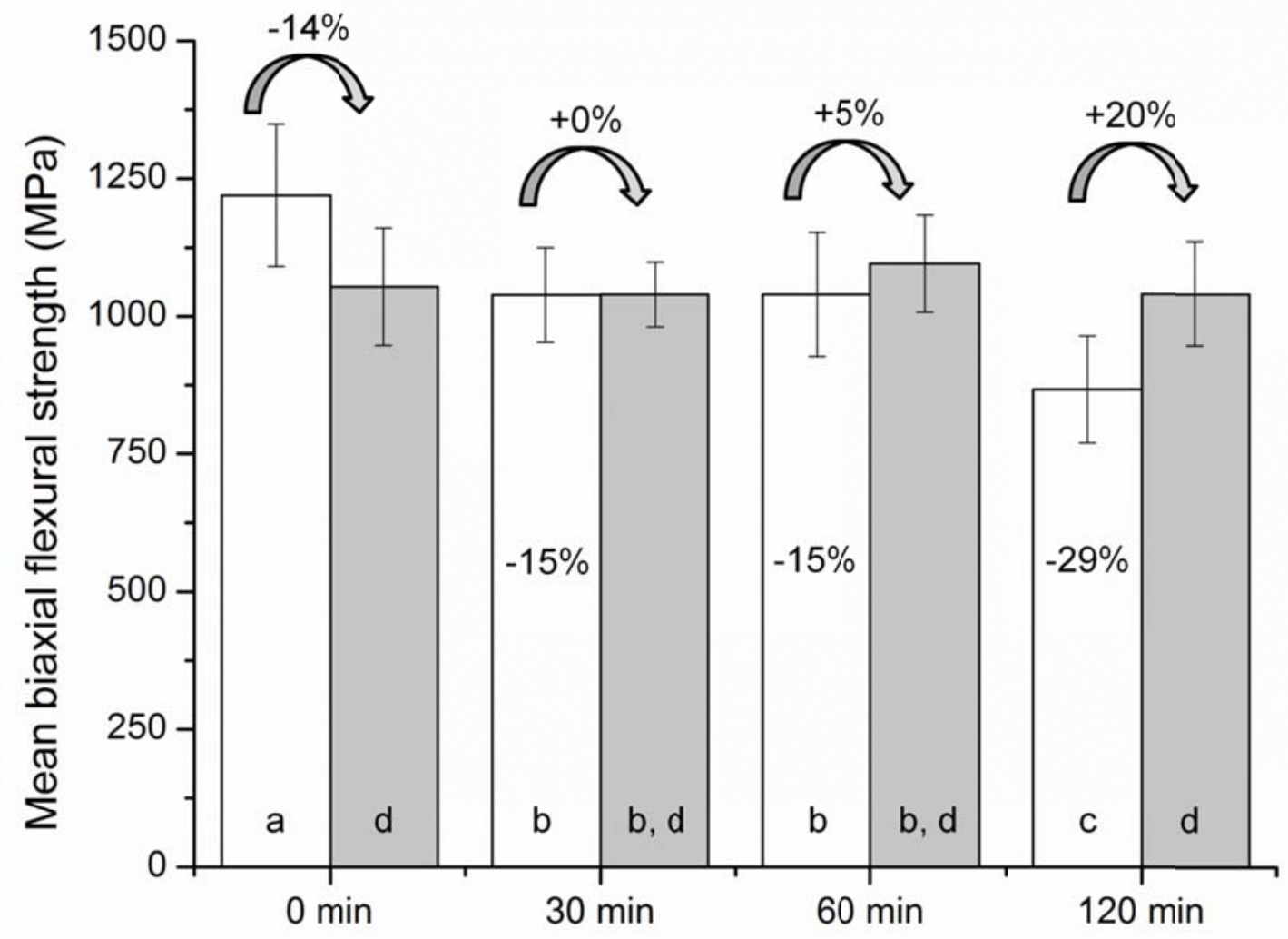

Figure 7. Mean biaxial flexural strength for different etching times. Values inside the columns indicate the relative variation with respect to the Control group. Values above the columns indicate the relative variation after ageing. Letters indicate the groups which are statistically similars. Error bars represent the standard deviations. 
The mean biaxial flexural strength results are shown Figure 7. The two-way ANOVA evidenced a main effect of etching time $(F(3,69)=10.272, p<0.001)$, no main effect of ageing $(F(1,69)=0.488, p=0.487)$ and an interaction between etching time and ageing, $(F(3,69)=$ $8.869, \mathrm{p}<0.001)$. The assumption of homogeneity of the variance was verified $(\mathrm{p}=0.356)$.

The results of the simple main effects analysis which indicate statistically significant differences between groups are represented by letters in Figure 7. In particular, they show that:

- All etched groups exhibited a significantly lower strength than the Control group and there was no significant difference between the HF40-30 and HF40-60 groups.

- There was no significant difference between any of the aged groups.

- The Control-Aged group exhibited a significantly lower strength than the Control group, there was no significant difference between respectively the HF40-30, HF40-60 and the HF40-30-Aged, HF40-60-Aged groups whereas the HF40-120-Aged group exhibited a significantly higher strength than the HF40-120 group.

Weibull analysis (Figure 8, Table 2) confirmed the decrease of strength associated to etching observed by the two-way ANOVA. The strength distributions of HF40-30 and HF40-60 groups were very similar. The decrease in strength was more pronounced for the HF40-120 group.

Regarding the effect of ageing, Weibull analysis confirmed that:

- The strength distributions of the all the aged groups were very similar.

- Ageing induced a decrease in strength for the control samples.

- Ageing induced an increase in strength for the samples etched two hours. 
Because of the limited number of samples, interpretations regarding the Weibull modulus are subjected to caution. It seems however that neither etching, nor ageing induced substantial changes in the Weibull modulus.

Table 2. Weibull modulus (biased), characteristic strengths and associated linear regression coefficients for different etching conditions, before and after ageing.

\begin{tabular}{|c|c|c|c|}
\hline Sample group & $\mathrm{m}(90 \%$ confidence interval $)$ & $\sigma_{0}[\mathrm{MPa}](90 \%$ confidence interval $)$ & $\mathrm{R}^{2}$ \\
\hline Control & $11(6-14)$ & $1278(1202-1362)$ & 0.9504 \\
\hline HF40-30 & $10(5-14)$ & $1064(994-1141)$ & 0.9483 \\
\hline HF40-60 & $8(4-11)$ & $1071(981-1173)$ & 0.9852 \\
\hline HF40-120 & $10(6-13)$ & $912(855-975)$ & 0.9167 \\
\hline Control-Aged & $10(6-14)$ & $1105(1039-1178)$ & 0.9591 \\
\hline HF40-30-Aged & $20(11-27)$ & $1068(1033-1103)$ & 0.9804 \\
\hline HF40-60-Aged & $13(7-17)$ & $1142(1085-1204)$ & 0.8779 \\
\hline HF40-120-Aged & $12(6-16)$ & $1081(1022-1145)$ & 0.9215 \\
\hline
\end{tabular}



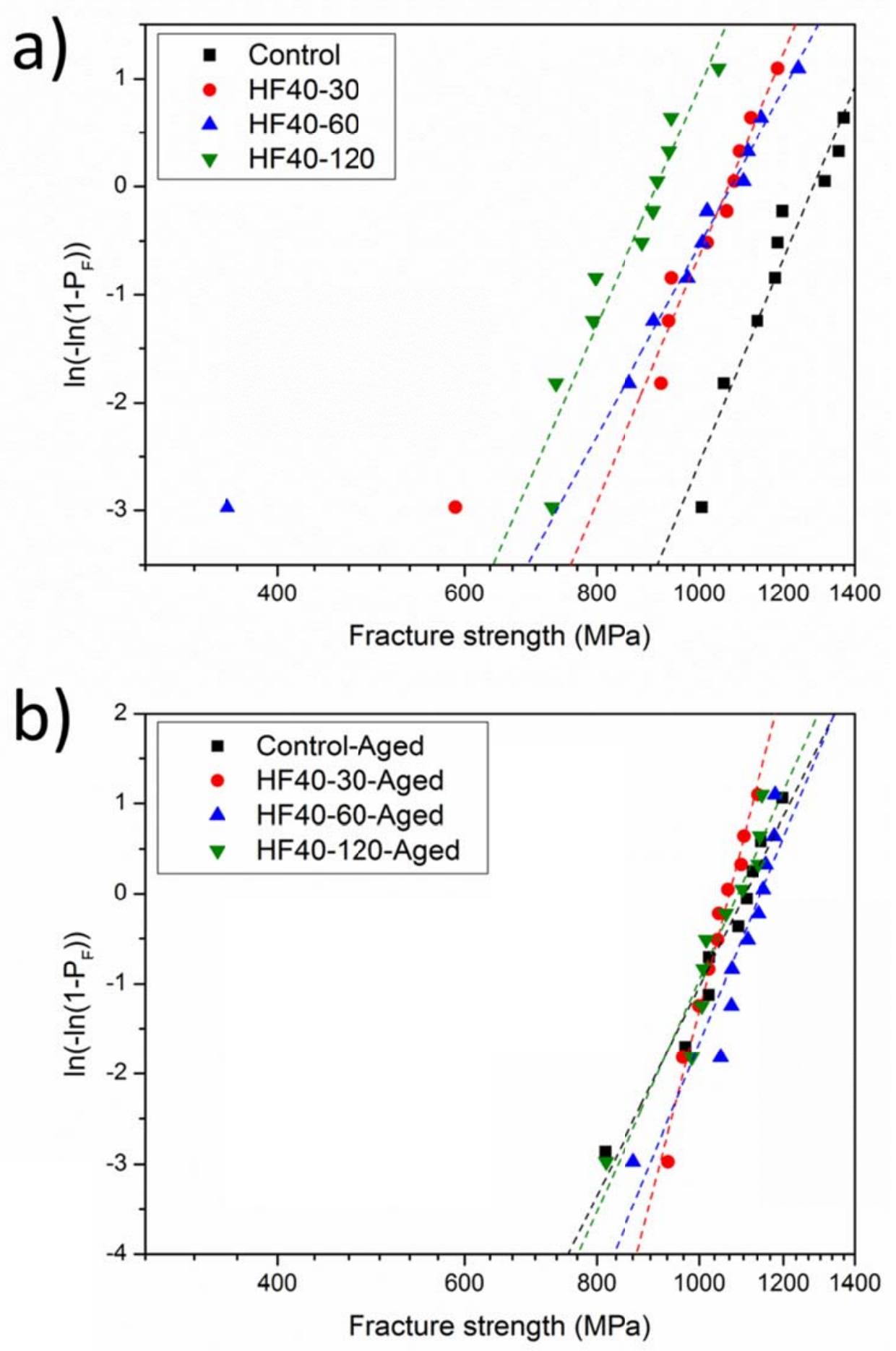

Figure 8. Weibull analysis of the fracture strength of 3Y-TZP before (a) and after ageing (b) for different etching times. 


\section{Discussion}

\subsection{Effects of HF etching}

\subsubsection{Qualitative interpretation}

HF etching induced an increase of monoclinic phase content at the surface. This could be explained by differences in the dissolution kinetics of yttrium oxide and zirconium oxide by HF, which could create an yttrium depleted layer of zirconia, in which the tetragonal phase is less stable.

Besides etching induced different kind of defects in the material:

- The formation of a thin etch-damaged layer on the whole surface, whose depth did not appear to evolve substantially over time, but could be locally thicker (Figure 2). Because the porosity was formed by dissolution, it is necessarily interconnected. The etchdamaged layer could thus be considered as micro-cracked. Besides, as commented before, etching induced some phase transformation. As a result, the fracture toughness of the surface is lower since it is that of monoclinic zirconia.

- Localized damage such as pits (Figure 3, Figure 1, Figure 6-b) or deep porous zones (Figure 6-a). This kind of flaws could result from the interaction of HF with pre-existing defects, such as pre-existing pores on the polished surface (Figure 3-a) or low densified regions. However the formation of pits could also be favored by a phenomenon of autocatalysis, in which the reaction products would increase the etch rate locally, as it has been observed for instance in silicon [20]. This would be consistent with the exponential relationship between mass loss and HF concentration which was previously reported [12]. 
- Additionally, etching could bring to the surface flaws which were just below by dissolving the material that separated them from the outside. For instance, even though the case was isolated, it is worthwhile recalling that the fracture of a sample from the HF40-30 group was associated to a "tunnel-like" defect (Figure 6-e). This defect probably existed previously but was shown up by etching.

As a consequence, etching induced a decrease in the average strength, without apparently affecting the strength variability. This decrease appeared to be much more important for the HF40-120 group (-29\%) than for the HF40-30 and the HF40-60 groups, for which the decrease $(-15 \%$ in both cases) and the Weibull strength distributions were similar. It seems thus recommendable not to use etching times superior to one hour, which moreover don't bring clear benefits in terms of roughness [12].

\subsubsection{Modeling}

To understand how the decrease of strength discussed above is related to the effect of etching on the population of superficial defects, some simple models are proposed below.

First of all, to estimate the size of the critical defect in each sample group, let us consider a hypothetical semi-circular surface crack of radius a. The stress intensity factor $\mathrm{K}_{\mathrm{I}}$ for an applied stress $\sigma_{\mathrm{A}}$ is :

$$
K_{I}=1.26 \times \sigma_{A} \sqrt{a}(7)
$$

To determine a, the knowledge of the fracture toughness of the material is needed. Unfortunately, because of the small grain size and of the phase transformation phenomenon, its measurement is challenging for $3 \mathrm{Y}$-TZP and the values reported in the literature differ 
substantially depending on the method used. Nevertheless measurement techniques involving a notch with a small tip radius appear more reliable, and the value retained here is $\mathrm{K}_{\mathrm{IC}}=4.1$ MPa.m ${ }^{1 / 2}$ [21]. Considering $\sigma_{\mathrm{A} 1}=1220 \mathrm{MPa}, \sigma_{\mathrm{A} 2}=1040 \mathrm{MPa}, \sigma_{\mathrm{A} 3}=1040 \mathrm{MPa}, \sigma_{\mathrm{A} 4}=870 \mathrm{MPa}$ which are respectively the mean strength of the Control, HF40-30, HF40-60 and HF-40-120 groups, the critical crack radii are $\mathrm{a}_{1} \approx 7 \mu \mathrm{m}, \mathrm{a}_{2} \approx 10 \mu \mathrm{m}, \mathrm{a}_{3} \approx 10 \mu \mathrm{m}, \mathrm{a}_{4} \approx 14 \mu \mathrm{m}$.

The etch-damaged layer, which could extend an existing surface flaw, can reach locally 3 $\mu \mathrm{m}$ (Figure 2). Therefore, according to this model, it could explain the increase of the critical defect size and thus the drop in strength observed for the HF40-30 and HF40-60 groups, but not for the HF40-120 group.

On the other hand, big pits were observed on the surface of samples from the HF40-120 groups and fractographic analysis evidenced that they can play a role in failure (Figure 1, Figure 6-b). As a simple model for these pits, let us consider a surface spherical pore of radius $\mathrm{R}$ under a constant biaxial tension $\sigma_{\mathrm{A}}$. For a Poisson ratio $\nu=0.3$, the maximum tensile stress $\sigma$ at a radial distance $r$ from the pore is given by [22]:

$$
\sigma=\sigma_{A}\left[1+1.091\left(1+\frac{r}{R}\right)^{-5}+9.091 \times 10^{-2}\left(1+\frac{r}{R}\right)^{-3}\right](8)
$$

Let us then consider then that this pore is associated to a circumferential crack (Figure 9-a, Figure 9-b). The stress intensity factor can be determined by the methodology proposed by Zimmermann et al. [22] which is briefly summarized here. 
a)

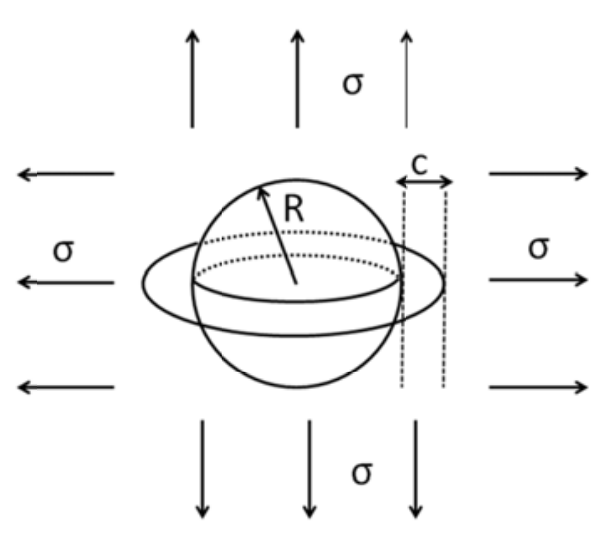

c)

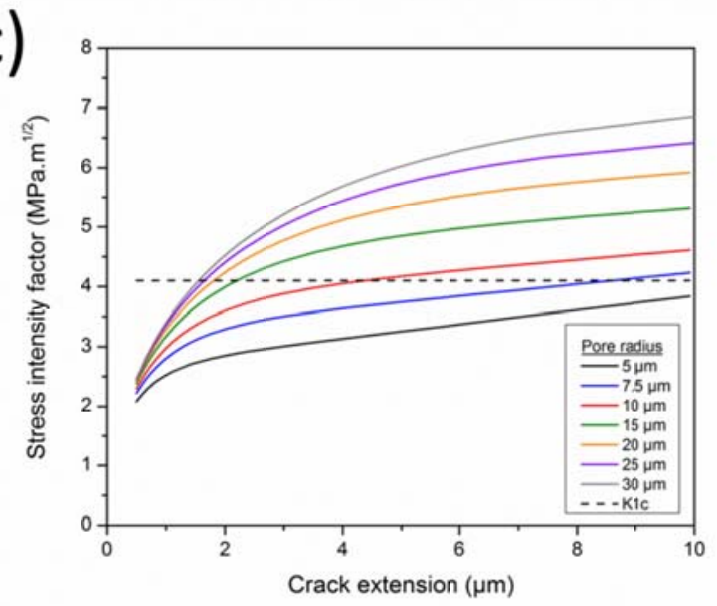

b)

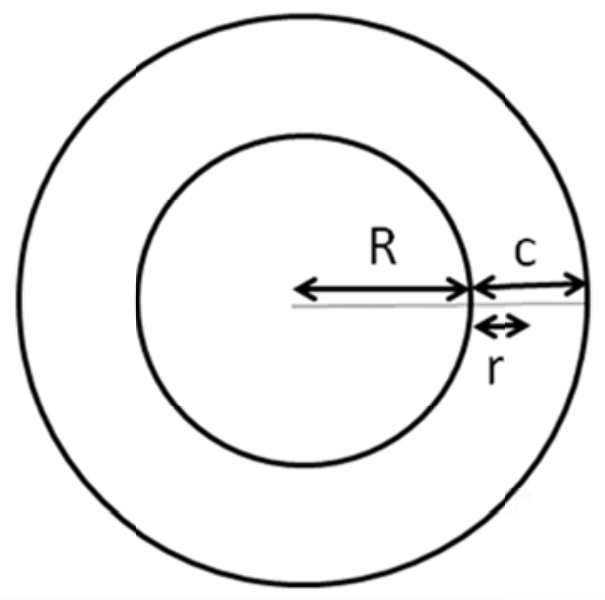

d)

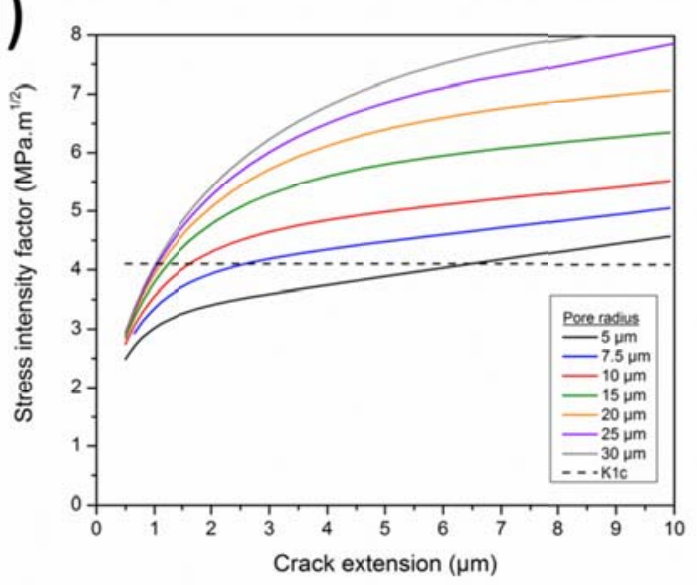

Figure 9. Top: (a) Schematic three-dimensional representation of a spherical pore with a circumferential crack under biaxial tension, (b) Schematic two-dimensional representation of an intersection through the crack plane. Bottom: theoretical stress intensity factor as function of crack extension for different pore radii and an applied biaxial stress of $870 \mathrm{MPa}$ (c) and 1040 $\operatorname{MPa}(d)$.

The calculation of the stress intensity factor $\mathrm{K}$ is based on:

$$
K=\int_{0}^{c} h(r, c) \sigma(r) d r(9)
$$


where $\mathrm{c}$ is the crack extension, $\sigma(\mathrm{r})$ can be determined from (8) and the weight function $\mathrm{h}$ is given by:

$$
h=\beta h_{\text {edge } \text { crack }}+(1-\beta) h_{\text {circular crack }}(10)
$$

where $h_{\text {edge crack }}$ and $h_{\text {circular crack }}$ are the weight functions of an edge crack and a circular crack and $\beta$ is an interpolation function.

$\beta, \mathrm{h}_{\text {edge crack }}$ and $\mathrm{h}_{\text {circular crack }}$ can be determined from the following formulas:

$$
\begin{gathered}
\beta=\frac{1}{\left(1+2 \frac{c}{R}\right)^{2}}(11) \\
h_{\text {edge crack }}=\frac{2}{(\pi c)^{1 / 2}} \frac{\left[1.3-0.71\left(\frac{r}{c}\right)^{2}+0.41\left(\frac{r}{c}\right)^{3}\right]}{\left[1-\left(\frac{r}{c}\right)^{2}\right]^{1 / 2}}(12) \\
h_{\text {circular crack }}=\frac{2}{[\pi(c+R)]^{\frac{1}{2}}} \frac{(r+R)}{\left[(c+R)^{2}-(r+R)^{2}\right]^{1 / 2}}(13)
\end{gathered}
$$

The software Maple (version 17, Maple Inc., Waterloo, Ontario, Canada) was used to calculate numerically the integral of equation (9) for different pore radii and a given applied tension as a function of crack extension. The curves obtained for $\sigma_{\mathrm{A}}=1040 \mathrm{MPa}$ and $\sigma_{\mathrm{A}}=870$ $\mathrm{MPa}$, which are respectively the mean flexural strength from both the HF40-30 and HF40-60 group and from the HF40-120 group are shown in Figure 9.

From these curves it can be seen that:

- For an applied tension $\sigma_{\mathrm{A}}=1040 \mathrm{MPa}$, pits of radius $\mathrm{R} \leq 5 \mu \mathrm{m}$ are unlikely to provoke failure because they should be associated to a crack of at least $7 \mu \mathrm{m}$, whereas pits of 
radius $\mathrm{R} \geq 7.5 \mu \mathrm{m}$ could become critical for small crack extensions: $\mathrm{c} \geq 2.6 \mu \mathrm{m}$ for $\mathrm{R}=$ $7.5 \mu \mathrm{m}, \mathrm{c} \geq 1.2 \mu \mathrm{m}$ for $\mathrm{R}=15 \mu \mathrm{m}, \mathrm{c} \geq 1 \mu \mathrm{m}$ for $\mathrm{R}=15 \mu \mathrm{m}$.

- For an applied tension $\sigma_{\mathrm{A}}=870 \mathrm{MPa}$, pits of radius $\mathrm{R} \leq 10 \mu \mathrm{m}$ are unlikely to provoke failure because they should be associated to a crack of at least $4.5 \mu \mathrm{m}$, whereas pits of radius $R \geq 15 \mu \mathrm{m}$ could become critical for small crack extensions: $\mathrm{c} \geq 2.2 \mu \mathrm{m}$ for $\mathrm{R}=15$ $\mu \mathrm{m}, \mathrm{c} \geq 1.6 \mu \mathrm{m}$ for $\mathrm{R}=30 \mu \mathrm{m}$.

Considering these results, the presence of big pits (Figure 1) associated to the cracks of the etch-damaged layer may explain the important decrease in strength of the HF40-120 group. The pits observed in the HF40-30 and in the HF40-60 specimens were smaller, which makes less probable their potential role in failure.

Finally, fractographic analysis also evidenced that deep porous zones could initiate fracture (Figure 6). The complicated geometry of this kind of defects makes modeling difficult. However, on the same manner as pits, they could be considered as stress concentrators associated to a crack.

To summarize, the models presented above tend to show that:

- For the HF40-30 and HF40-60 groups in which the pits are smaller than in the HF40-120 group, the model of an existing crack whose dimension is increased by the etch-damaged layer gives a satisfying explanation of the drop in strength induced by etching. The fact that the thickness of the etch-damaged layer was not related to etching time supports this hypothesis, because it explains the similarity between the HF40-30 and HF40-60 strength distributions. 
- For the HF40-120 group, the presence of big pits associated to the etch-damaged layer and of deep porous zones explains the more important drop in strength.

\subsection{Effects of ageing}

\subsubsection{Qualitative interpretation}

There was no substantial difference in the amount of monoclinic phase between the different groups of etched-aged specimens. Another observation worthwhile recalling is that the increase of monoclinic content associated to ageing in the etched samples was close to the increase observed in the Control-Aged group. Furthermore it was observed on FIB cross-sections that the thickness of the micro-cracked layer induced by phase transformation during ageing was not more important in a sample etched for two hours than in the control one. Accordingly the influence of previous etching on ageing appears to be limited.

Moreover, there was surprisingly no significant difference in the strength distributions among all the specimen groups after ageing (see Figure 7 and Figure 8). The average strength of all etched groups remained constant or increased after ageing, whereas that of the samples from the Control-Aged group decreased. This opposite behavior must be related to a distinct effect of ageing on control and on etched specimens, or to two competing mechanisms, one of them tending to neutralize etching defects.

The drop in strength induced by ageing of polished zirconia has already been reported in numerous studies [23-27]. It has been suggested to be caused by the apparition of micro-cracks due to the phase transformation and by the depletion of transformable tetragonal phase on the surface. The fractographic observations presented in this work bring an additional element: they 
show that failure can be initiated in regions in which the micro-cracked layer induced by ageing is locally thicker (Figure 6-c).

On the other hand, the increase in strength subsequent to ageing etched specimens has never been reported. At first, two hypotheses appear to be reasonable to explain this phenomenon. One is that ageing of etched specimens could induce a change in the critical defects responsible for fracture, and the other is that ageing may induce a change in the stress distribution at the surface of etched specimens. However, this second hypothesis appears to be unlikely, since there is no apparent reason for which the presence of a residual compressive stress on the surface would induce an increase in strength for etched specimens but not for control ones.

On the contrary, the effect of ageing on the critical defects could explain the differences observed, as it will be demonstrated in the next section. Indeed, a critical flaw induced by etching is necessarily connected to the surface and is therefore necessarily under direct exposure of water vapor. The tip of the crack could thus be transformed, reducing its sharpness. A similar phenomenon of crack tip blunting was suggested to exist by Marro et al. [28], who found a large increase of strength after ageing pre-indented zirconia bars.

\subsubsection{Modeling}

\subsubsection{Strength decrease}

To explain the drop in strength induced by ageing in the Control-Aged group, let us consider first the case of the sample shown in Figure 6-c, for which the depth of the micro-cracked layer induced by ageing reached locally $\mathrm{d} \approx 13 \mu \mathrm{m}$ and broke at $\sigma_{\mathrm{A}}=970 \mathrm{MPa}$. The radius of an equivalent semi-circular crack which would provoke a failure at this stress is a $\approx 11 \mu \mathrm{m}$, therefore the dimensions of this flaw are close to that of the theoretical critical defect. 
On the other hand, let us consider the case that the degraded layer thickness is homogenous ( $d \approx 5 \mu \mathrm{m})$. It has been suggested that the minimum value of $d$ necessary for a crack to continue propagating through the tetragonal bulk can be estimated from [27]:

$$
d_{\text {min }} \approx 0.49 \times a_{c}(14)
$$

where $a_{c}$ is the critical defect size in the material before ageing. As shown previously, in the case of the Control group $\mathrm{a}_{\mathrm{c}} \approx 7 \mu \mathrm{m}$, therefore $\mathrm{d}>>\mathrm{d}_{\min }\left(\mathrm{d}_{\min } \approx 3.5 \mu \mathrm{m}\right)$.

These two observations tend to demonstrate that the strength of the Control-Aged samples is governed by the aged damaged layer, which is responsible for the drop in strength, and not by the pre-existing flaws. The mechanism of crack tip blunting discussed below has thus no effect in this case.

\subsubsection{Strength increase}

To explain how pre-existing etching defects could be neutralized and how it could lead to strength increase for the HF40-120 samples, let us consider the hypothesis that ageing could induce some crack tip blunting. Modeling the case of a cracked pit is not straightforward. Therefore, to get an order of magnitude of the blunting necessary to explain the strength increase observed, let us consider the relationship between the stress intensity factors at fracture of a blunt and a sharp crack [29]:

$$
\frac{K_{I C, \text { blunt }}}{K_{I C}}=\left(1+\frac{\rho_{0}}{2 r_{0}}\right)^{1 / 2}(15)
$$

where $\mathrm{K}_{\mathrm{IC} \text {,blunt }}$ is the stress intensity factor of the blunt crack at fracture, $\mathrm{K}_{\mathrm{IC}}$ is the fracture toughness, $\rho_{0}$ is the root radius of the blunt crack and $\mathrm{r}_{0}=\mathrm{w} \sim 2 \mathrm{w}$ with $\mathrm{w}$ an effective grain width at the crack tip. Assuming that $\mathrm{w} \approx 0.3 \mu \mathrm{m}$ (grain size of the $3 \mathrm{Y}$-TZP used in this work measured 
by the intercept method) and that the relationship between the strength of the HF40-120-aged (blunt crack) and HF40-120 (sharp crack) specimens corresponds to the relationship of eq. (15), we obtain $\rho_{0}=0.3 \sim 0.6 \mu \mathrm{m}$, which shows that a blunting of the crack tip to a radius of the order of magnitude of the grain size could explain the strength increase.

\subsubsection{Summary}

Ageing seems to lead to two competing mechanisms:

- On the one hand, etching defects, which are directly accessible to water vapor, are neutralized: a moderate blunting of the crack tip is sufficient to explain the strength increase observed for the HF40-120 samples.

- On the other hand, the apparition of the degraded layer explains the drop in strength for the control samples. Considering that etching defects are fully inactivated, the strength will be also governed by the degraded layer in the etched-aged groups, which explains the similarity between all the strength distributions after ageing.

\section{Conclusion}

Etching of $3 \mathrm{Y}-\mathrm{TZP}$ with HF $40 \%$ for times up to two hours has been studied in terms of surface integrity, flexural strength and resistance to hydrothermal degradation. It is concluded that etching induces an increase in monoclinic phase content and a decrease in the biaxial flexural strength of polished specimens. In both cases, these changes do not exceed $15 \%$ for etching times below one hour. Besides, HF etched zirconia apparently does not exhibit an increased sensitivity to LTD and the biaxial strength of etched specimens can even increase with ageing, which can be explained in terms of crack tip blunting by phase transformation. 
Accordingly, within the limits of this study, dental zirconia etched with HF $40 \%$ should be reliable for long-term implantations provided that the etching duration does not exceed one hour.

\section{Acknowledgement}

The authors would like to acknowledge the European Commission funding under the 7th Framework Programme (Marie Curie Initial Training Networks; grant number: 289958, Bioceramics for bone repair), the support of the Ministry of Economy and Competitiveness (MINECO) of Spain (project ref. MAT2011-23913) and the Government of Catalonia for the grant 2014SGR0137. The authors would also like to thank Dr. Trifon Trifonov for his help during FIB/SEM sessions.

\section{Abbreviations}

DI water, Deionized water; FIB, Focused Ion Beam; HF, Hydrofluoric acid; LTD, Low Temperature Degradation; SEM, Scanning Electron Microscopy; Y-TZP, Yttria-stabilized tetragonal zirconia polycrystals; 3 Y-TZP, $3 \mathrm{~mol} \%$ Y-TZP.

\section{References}

[1] Chevalier J. What future for zirconia as a biomaterial? Biomaterials 2006;27:535-43. doi:10.1016/j.biomaterials.2005.07.034.

[2] Garvie R, Hannink R, Pascoe R. Ceramic steel? Nature 1975;258:703-4.

[3] Lawson S. Environmental degradation of zirconia ceramics. J Eur Ceram Soc 1995;15:485-502. doi:10.1016/0955-2219(95)00035-S.

[4] Denry I, Kelly JR. State of the art of zirconia for dental applications. Dent Mater 2008;24:299-307. doi:10.1016/j.dental.2007.05.007.

[5] Andreiotelli M, Kohal R-J. Fracture strength of zirconia implants after artificial aging. Clin Implant Dent Relat Res 2009;11:158-66. doi:10.1111/j.1708-8208.2008.00105.x. 
[6] Osman RB, Swain M V, Atieh M, Ma S, Duncan W. Ceramic implants (Y-TZP): are they a viable alternative to titanium implants for the support of overdentures? A randomized clinical trial. Clin Oral Implants Res 2014;25:1366-77. doi:10.1111/clr.12272.

[7] Kohal R-J, Knauf M, Larsson B, Sahlin H, Butz F. One-piece zirconia oral implants: oneyear results from a prospective cohort study. 1. Single tooth replacement. J Clin Periodontol 2012;39:590-7. doi:10.1111/j.1600-051X.2012.01876.x.

[8] Wennerberg A, Albrektsson T. On implant surfaces: a review of current knowledge and opinions. Int J Oral Maxillofac Implants 2009;25:63-74.

[9] Gahlert M, Röhling S, Wieland M, Eichhorn S, Küchenhoff H, Kniha H. A comparison study of the osseointegration of zirconia and titanium dental implants. A biomechanical evaluation in the maxilla of pigs. Clin Implant Dent Relat Res 2010;12:297-305. doi:10.1111/j.1708-8208.2009.00168.x.

[10] Ito H, Sasaki H, Saito K, Honma S, Yajima Y, Yoshinari M. Response of osteoblast-like cells to zirconia with different surface topography. Dent Mater J 2013;32:122-9. doi:10.4012/dmj.2012-208.

[11] Bergemann C, Duske K, Nebe JB, Schöne A, Bulnheim U, Seitz H, et al. Microstructured zirconia surfaces modulate osteogenic marker genes in human primary osteoblasts. J Mater Sci Mater Med 2015. doi:10.1007/s10856-014-5350-x.

[12] Flamant Q, García Marro F, Roa Rovira JJ, Anglada M. Hydrofluoric acid etching of dental zirconia. Part 1: etching mechanism and surface characterization. n.d.

[13] Oliva J, Oliva X, Oliva JD. Five-year success rate of 831 consecutively placed Zirconia dental implants in humans: a comparison of three different rough surfaces. Int $J$ Oral Maxillofac Implants 2010;25:336-44.

[14] Sanon C, Chevalier J, Douillard T, Cattani-Lorente M, Scherrer SS, Gremillard L. A new testing protocol for zirconia dental implants. Dent Mater 2015;31:15-25. doi:10.1016/j.dental.2014.09.002.

[15] Chevalier J, Cales B, Drouin JM. Low-temperature aging of Y-TZP ceramics. J Am Ceram Soc 1999;82:2150-4.

[16] Toraya H, Yoshimura M, Somiya S. Calibration Curve for Quantitative Analysis of the Monoclinic-Tetragonal ZrO2 System by X-Ray Diffraction. J Am Ceram Soc 1984;67:C $119-\mathrm{C}-121$.

[17] Börger A, Supancic P, Danzer R. The ball on three balls test for strength testing of brittle discs: stress distribution in the disc. J Eur Ceram Soc 2002;22:1425-36. doi:10.1016/S0955-2219(01)00458-7. 
[18] Shah K, Holloway J a, Denry IL. Effect of coloring with various metal oxides on the microstructure, color, and flexural strength of 3Y-TZP. J Biomed Mater Res B Appl Biomater 2008;87:329-37. doi:10.1002/jbm.b.31107.

[19] Munz D, Fett T. Ceramics: mechanical properties, failure behaviour, materials selection. vol. 36. Springer Science \& Business Media; 1999.

[20] Shah IA, van der Wolf BMA, van Enckevort WJP, Vlieg E. Wet Chemical Etching of Silicon \{111\}: Autocatalysis in Pit Formation. J Electrochem Soc 2008;155:J79. doi:10.1149/1.2830841.

[21] Turon-vinas M, Anglada M. Fracture toughness of zirconia from a shallow notch produced by ultra-short pulsed laser ablation. J Eur Ceram Soc 2014;34:3865-70. doi:10.1016/j.jeurceramsoc.2014.05.009.

[22] Zimmermann A, Rodel J. Generalized Orowan-Petch Plot for Brittle Fracture. J Am Ceram Soc 1998;81:2527-32. doi:10.1111/j.1151-2916.1998.tb02657.x.

[23] Drummond JL. In Vitro Aging of Yttria-Stabilized Zirconia. J Am Ceram Soc 1989;72:675-6. doi:10.1111/j.1151-2916.1989.tb06194.x.

[24] Ardlin BI. Transformation-toughened zirconia for dental inlays, crowns and bridges: Chemical stability and effect of low-temperature aging on flexural strength and surface structure. Dent Mater 2002;18:590-5. doi:10.1016/S0109-5641(01)00095-1.

[25] Kim H-T, Han J, Yang J, Lee J, Kim S. The effect of low temperature aging on the mechanical property \& phase stability of Y-TZP ceramics. J Adv Prosthodont 2009;1:113-7. doi:10.4047/jap.2009.1.3.113.

[26] Kohorst P, Borchers L, Strempel J, Stiesch M, Hassel T, Bach F-W, et al. Lowtemperature degradation of different zirconia ceramics for dental applications. Acta Biomater 2012;8:1213-20. doi:10.1016/j.actbio.2011.11.016.

[27] Marro FG, Mestra a., Anglada M. Weibull strength statistics of hydrothermally aged 3 mol\% yttria-stabilised tetragonal zirconia. Ceram Int 2014;40:12777-82. doi:10.1016/j.ceramint.2014.04.131.

[28] Marro FG, Anglada M. Strengthening of Vickers indented 3Y-TZP by hydrothermal ageing. J Eur Ceram Soc 2012;32:317-24. doi:10.1016/j.jeurceramsoc.2011.08.019.

[29] Deng Z-Y, She J, Inagaki Y, Yang J-F, Ohji T, Tanaka Y. Reinforcement by crack-tip blunting in porous ceramics. J Eur Ceram Soc 2004;24:2055-9. doi:10.1016/S09552219(03)00365-0. 